Supplement of Ann. Geophys., 38, 123-135, 2020

https://doi.org/10.5194/angeo-38-123-2020-supplement

(C) Author(s) 2020. This work is distributed under

the Creative Commons Attribution 4.0 License.

(c) (1)

Supplement of

\title{
Magnetospheric Multiscale observations of energetic oxygen ions at the duskside magnetopause during intense substorms
}

\section{Chen Zeng et al.}

Correspondence to: Chi Wang (cw@ spaceweather.ac.cn) and Suping Duan (spduan@nssc.ac.cn)

The copyright of individual parts of the supplement might differ from the CC BY 4.0 License. 


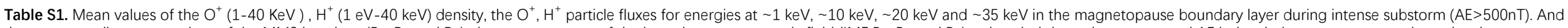
the corresponding mean values of the MMS locations (Rx, Ry and Rz), three components of the interplanetary magnetic field (IMF Bx, By and Bz), solar wind dynamic pressure and AE index during magnetopause boundary layer

crossings. The 0 and 1 indicate the events occured during the expansion phase and recovery phase of intense substorm, respectively.

\begin{tabular}{|c|c|c|c|c|c|c|c|c|c|c|c|c|c|c|c|c|c|c|c|}
\hline \multirow{2}{*}{$\begin{array}{l}\text { Magnetopause boundary } \\
\text { layer crossing time } \\
\text { interval }\end{array}$} & \multirow{2}{*}{$\begin{array}{c}\mathrm{N}_{\mathrm{O}^{+}} \\
\left(\mathrm{cm}^{-3}\right) \\
(1-40 \mathrm{keV})(1\end{array}$} & \multirow{2}{*}{$\begin{array}{c}\mathrm{N}_{\mathrm{H}^{+}} \\
\left(\mathrm{cm}^{-3}\right) \\
\mathrm{eV}-40 \mathrm{keV})\end{array}$} & \multirow{2}{*}{$\begin{array}{c}\mathrm{O}^{+} \text {Flux } \\
1 \mathrm{keV}\end{array}$} & \multirow{2}{*}{$\begin{array}{c}\mathrm{H}^{+} \text {Flux } \\
1 \mathrm{keV}\end{array}$} & \multirow{2}{*}{$\begin{array}{l}\mathrm{O}^{+} \text {Flux } \\
10 \mathrm{keV}\end{array}$} & $\mathrm{H}^{+}$Flux & $\mathrm{O}^{+}$Flux & \multirow{2}{*}{$\begin{array}{l}\mathrm{H}^{+} \text {Flux } \\
20 \mathrm{keV}\end{array}$} & \multirow{2}{*}{$\begin{array}{l}\mathrm{O}^{+} \text {Flux } \\
35 \mathrm{keV}\end{array}$} & \multirow{2}{*}{$\begin{array}{l}\mathrm{H}^{+} \text {Flux } \\
35 \mathrm{keV}\end{array}$} & \multicolumn{3}{|c|}{ Position $\left(R_{E}\right)$} & \multicolumn{3}{|c|}{$\operatorname{IMF}(\mathrm{nT})$} & \multirow{2}{*}{$\begin{array}{l}\text { Psw } \\
(\mathrm{nPa})\end{array}$} & \multirow{2}{*}{$\begin{array}{l}\mathrm{AE} \\
(\mathrm{nT})\end{array}$} & \multirow{2}{*}{ Phase } \\
\hline & & & & & & \multicolumn{2}{|c|}{ Flux $\left(\mathrm{cm}^{2} \mathrm{~s} \mathrm{sr} \mathrm{eV}\right)^{-1}$} & & & & $\mathrm{Rx}$ & Ry & Rz & $\mathrm{Bx}$ & By & $\mathrm{Bz}$ & & & \\
\hline 2015-09-07/07:05-07:10 & 0.142 & 1.237 & 37.230 & 274.289 & 42.911 & 725.507 & 21.682 & 601.114 & 6.157 & 459.269 & 4.786 & 4.033 & -1.749 & -3.089 & 4.256 & -9.451 & 2.205 & 629.545 & 1 \\
\hline 2015-09-07/17:55-18:05 & 0.091 & 11.613 & 35.929 & $29,934.206$ & 15.230 & 586.124 & 12.633 & 230.363 & 4.931 & 149.602 & 1.574 & 10.879 & -4.372 & 1.612 & 3.605 & -8.888 & 3.528 & 941.545 & 1 \\
\hline 2015-09-07/20:55-21:05 & 0.046 & 3.080 & 31.220 & $7,061.769$ & 6.990 & 79.934 & 2.187 & 21.917 & 0.848 & 5.694 & -0.197 & 10.206 & -3.186 & -0.145 & 6.852 & -8.408 & 4.132 & 835.909 & 0 \\
\hline 2015-09-09/08:35-08:45 & 0.599 & 14.406 & 179.469 & $42,735.070$ & 76.019 & 496.719 & 29.063 & 251.542 & 6.214 & 149.610 & 5.276 & 5.971 & -3.318 & 1.373 & 1.760 & -7.910 & 3.937 & 606.364 & 1 \\
\hline 2015-09-09/12:39-12:45 & 0.107 & 7.842 & 31.637 & $16,483.012$ & 17.698 & 564.204 & 9.887 & 325.903 & 2.622 & 177.350 & 4.423 & 8.870 & -5.536 & -0.763 & -4.249 & -7.456 & 2.322 & 741.714 & 0 \\
\hline 2015-09-09/13:15-13:25 & 0.056 & 14.047 & 37.296 & $21,678.069$ & 10.373 & 400.182 & 9.180 & 261.400 & 2.946 & 128.748 & 4.174 & 9.219 & -5.621 & -1.215 & -3.233 & -7.225 & 1.959 & 637.091 & 1 \\
\hline 2015-09-09/13:45-13:55 & 0.064 & 9.041 & 36.773 & $11,580.033$ & 12.045 & 306.715 & 6.542 & 137.802 & 1.541 & 50.572 & 3.981 & 9.458 & -5.636 & -1.345 & -2.694 & -7.194 & 2.721 & $1,038.273$ & 1 \\
\hline 2015-09-09/14:25-14:35 & 0.043 & 10.241 & 38.495 & $18,815.151$ & 8.119 & 375.745 & 4.932 & 222.822 & 1.523 & 124.868 & 3.661 & 9.802 & -5.586 & -1.339 & -1.984 & -6.619 & 3.493 & $1,139.000$ & 0 \\
\hline 2015-09-09/15:35-15:43 & 0.059 & 14.944 & 32.768 & $26,134.293$ & 11.508 & 377.701 & 8.116 & 236.275 & 2.112 & 144.860 & 3.097 & 10.279 & -5.335 & -3.020 & 0.044 & -6.352 & 2.803 & $1,005.556$ & 1 \\
\hline 2015-09-09/18:40-18:50 & 0.040 & 5.551 & 29.022 & $12,149.427$ & 8.418 & 553.067 & 4.270 & 240.704 & 1.273 & 87.097 & 1.367 & 10.756 & -4.066 & -2.744 & -1.611 & -6.038 & 3.342 & 856.545 & 0 \\
\hline 2015-09-10/19:10-19:20 & 0.046 & 20.640 & 37.101 & $22,002.970$ & 7.806 & 378.786 & 3.024 & 309.229 & 0.979 & 230.639 & 1.186 & 10.628 & -3.867 & 7.841 & -9.592 & -5.740 & 8.139 & 869.000 & 1 \\
\hline 2015-09-11/09:55-10:05 & 0.328 & 9.543 & 97.676 & $22,705.922$ & 33.864 & 597.728 & 12.118 & 382.099 & 3.356 & 258.424 & 5.403 & 7.128 & -4.424 & -5.303 & 3.485 & -5.553 & 5.864 & 689.364 & 1 \\
\hline 2015-09-14/10:05-10:15 & 0.049 & 9.756 & 42.821 & $23,447.125$ & 9.140 & 320.256 & 3.622 & 173.490 & 1.014 & 100.230 & 5.727 & 7.250 & -4.631 & -2.813 & 7.955 & -5.338 & 3.104 & 542.727 & 0 \\
\hline 2015-09-14/15:27-15:40 & 0.025 & 11.241 & 37.334 & $26,970.368$ & 4.851 & 363.691 & 2.780 & 177.241 & 1.334 & 82.722 & 3.760 & 10.031 & -5.403 & -4.193 & 1.985 & -5.086 & 1.990 & $1,048.786$ & 0 \\
\hline $2015-09-18 / 08: 30-08: 35$ & 0.045 & 16.843 & 40.623 & $18,853.343$ & 9.521 & 264.591 & 4.352 & 164.118 & 0.978 & 99.159 & 6.314 & 6.066 & -3.600 & 0.493 & 3.490 & -5.050 & 3.223 & 602.667 & 0 \\
\hline 2015-09-19/07:43-07:48 & 0.054 & 17.642 & 62.590 & $43,832.709$ & 9.269 & 521.361 & 3.712 & 396.036 & 1.121 & 227.670 & 6.344 & 5.414 & -2.997 & 2.262 & -7.480 & -4.958 & 4.010 & 851.833 & 0 \\
\hline 2015-09-20/06:35-06:45 & 0.102 & 14.666 & 80.937 & $35,712.553$ & 10.240 & $1,163.906$ & 7.678 & 602.357 & 2.772 & 325.489 & 6.154 & 4.352 & -2.088 & -9.394 & 6.299 & -4.925 & 7.106 & 752.091 & 0 \\
\hline $2015-09-20 / 11: 55-12: 10$ & 0.434 & 7.442 & 80.124 & $16,341.032$ & 71.086 & 400.462 & 28.091 & 240.984 & 6.978 & 143.438 & 5.999 & 8.204 & -5.510 & -5.784 & 5.480 & -4.753 & 2.717 & 521.188 & 0 \\
\hline 2015-09-20/13:08-13:15 & 0.016 & 10.544 & 30.954 & $13,836.314$ & 6.678 & 73.363 & 2.834 & 31.369 & 0.886 & 15.220 & 5.589 & 8.763 & -5.685 & -8.054 & 1.125 & -4.638 & 2.098 & 722.750 & 0 \\
\hline 2015-09-20/15:10-15:30 & 0.096 & 5.629 & 33.507 & $16,883.659$ & 15.966 & 248.980 & 13.872 & 116.136 & 4.277 & 81.692 & 4.632 & 9.612 & -5.452 & -6.761 & 2.044 & -4.557 & 1.932 & 904.381 & 0 \\
\hline 2015-09-20/15:50-16:00 & 0.060 & 6.365 & 31.734 & $13,611.068$ & 13.464 & 271.933 & 8.134 & 136.969 & 2.675 & 88.419 & 4.272 & 9.815 & -5.250 & -6.335 & 2.445 & -4.505 & 2.251 & 797.455 & 1 \\
\hline 2015-09-23/09:30-09:40 & 0.029 & 9.385 & 38.675 & $21,243.013$ & 4.903 & 309.771 & 2.772 & 95.593 & 1.145 & 43.088 & 6.868 & 6.745 & -4.430 & 7.346 & -1.930 & -4.083 & 2.180 & 578.000 & 0 \\
\hline 2015-10-03/07:20-07:35 & 0.085 & 8.665 & 36.580 & $17,494.913$ & 13.027 & 347.510 & 4.431 & 224.319 & 1.304 & 114.122 & 7.924 & 5.148 & -3.039 & -6.693 & 6.429 & -4.080 & 1.711 & 530.188 & 0 \\
\hline 2015-10-03/15:30-15:35 & 0.018 & 15.300 & 42.059 & $29,640.560$ & 4.033 & 420.373 & 2.519 & 229.615 & 0.974 & 104.451 & 5.831 & 8.840 & -5.035 & -4.737 & -3.845 & -3.942 & 2.385 & 775.500 & 1 \\
\hline 2015-10-04/09:20-09:30 & 0.059 & 3.884 & 29.528 & $9,586.658$ & 16.949 & 123.538 & 3.718 & 43.133 & 1.314 & 13.713 & 8.147 & 6.287 & -4.297 & -7.468 & 5.159 & -3.925 & 0.994 & 551.818 & 1 \\
\hline $2015-10-08 / 16: 38-16: 42$ & 0.035 & 4.557 & 28.378 & $11,347.393$ & 6.925 & 813.632 & 6.872 & 146.593 & 3.493 & 54.523 & 5.487 & 8.618 & -4.415 & -0.558 & -0.594 & -3.753 & 2.023 & 697.600 & 0 \\
\hline
\end{tabular}




\begin{tabular}{|c|c|c|c|c|c|c|c|c|c|c|c|c|c|c|c|c|c|c|}
\hline $15-10-09 / 15: 05-15: 10$ & 0.019 & 4.821 & 28.371 & $11,498.846$ & 4.675 & 14.226 & 1.982 & 12.652 & 0.769 & 103.451 & 6.614 & 8.263 & -4.885 & -4.038 & -2.435 & -3.748 & 2.116 & 953.833 \\
\hline $15-10-10 / 07: 10-07: 15$ & 0.022 & 2.286 & 33.746 & $6,406.791$ & 4.691 & 228.993 & 1.733 & 135.324 & 0.981 & 71.948 & 8.680 & 4.768 & -2.840 & -5.092 & 0.693 & -3.577 & 0.923 & 780.667 \\
\hline $15-10-14 / 13: 20-13: 25$ & 0.015 & 4.510 & 27.982 & $9,757.235$ & 4.640 & 135.480 & 1.699 & 73.304 & 0.789 & 35.535 & 8.026 & 7.321 & -4.898 & -2.128 & -0.328 & -3.520 & 1.980 & 516.333 \\
\hline $15-10-18 / 15: 05-15: 10$ & 0.197 & 7.830 & 48.944 & $21,293.363$ & 32.270 & 22.094 & 14.575 & 181.302 & 2.758 & 71.246 & 7.280 & 7.500 & -4.400 & -3.192 & 1.093 & -3.458 & 1.998 & 615.667 \\
\hline $15-11-08 / 14: 20-14: 25$ & 0.093 & 4.278 & 38.996 & $14,081.292$ & 14.963 & 129.709 & 9.067 & 71.091 & 3.005 & 36.468 & 8.552 & 5.343 & -3.136 & -6.512 & 6.132 & -3.220 & 1.213 & 624.167 \\
\hline $15-11-16 / 14: 20-14: 25$ & 0.179 & 10.410 & 69.184 & $38,612.681$ & 23.719 & 222.091 & 8.483 & 125.696 & 1.865 & 65.743 & 8.490 & 4.580 & -2.588 & -3.225 & 3.158 & -3.075 & 1.458 & 873.667 \\
\hline $16-09-26 / 13: 40-13: 45$ & 0.035 & 4.052 & 32.605 & $9,870.575$ & 5.268 & 165.454 & 1.733 & 111.824 & 0.829 & 56.191 & 3.195 & 7.324 & -5.318 & -4.700 & 4.693 & -2.880 & 3.153 & 517.500 \\
\hline $16-09-28 / 14: 47-14: 52$ & 0.132 & 2.220 & 35.432 & $6,675.883$ & 28.059 & 320.423 & 19.565 & 211.106 & 6.172 & 169.569 & 2.981 & 8.561 & -5.387 & -2.267 & 4.780 & -2.842 & 4.364 & $1,304.667$ \\
\hline $16-09-28 / 21: 15-21: 20$ & 0.077 & 1.753 & 43.496 & $3,805.188$ & 9.287 & 112.467 & 4.182 & 73.255 & 1.516 & 44.468 & -0.244 & 11.499 & -2.821 & -4.660 & 4.937 & -2.667 & 3.760 & 613.667 \\
\hline $16-09-29 / 16: 10-16: 15$ & 0.009 & 2.720 & 38.925 & $6,683.875$ & 5.114 & 159.368 & 2.688 & 78.244 & 1.808 & 49.182 & 2.494 & 9.773 & -5.082 & -5.688 & 0.307 & -2.359 & 2.123 & 832.667 \\
\hline $16-09-30 / 18: 15-18: 20$ & 0.007 & 1.568 & 25.038 & $4,772.589$ & 4.238 & 162.619 & 1.574 & 78.375 & 0.727 & 35.685 & 1.602 & 11.027 & -4.141 & 0.817 & 4.380 & -2.348 & 2.217 & $1,034.667$ \\
\hline $16-10-01 / 19: 00-19: 05$ & 0.008 & 4.288 & 31.446 & $9,139.342$ & 3.659 & 111.740 & 1.563 & 73.806 & 0.773 & 53.333 & 1.344 & 11.294 & -3.720 & -4.800 & 3.188 & -2.095 & 1.950 & 818.333 \\
\hline $16-10-02 / 19: 45-19: 50$ & 0.007 & 2.386 & 26.212 & $5,245.227$ & 3.428 & 307.222 & 1.602 & 176.983 & 0.862 & 92.688 & 1.076 & 11.445 & -3.302 & -2.505 & 0.973 & -1.883 & 2.417 & 601.000 \\
\hline $16-10-02 / 20: 40-20: 45$ & 0.009 & 0.992 & 27.296 & $2,920.964$ & 4.557 & 153.313 & 1.936 & 61.723 & 0.889 & 24.041 & 0.581 & 11.491 & -2.882 & -3.963 & 4.312 & -1.826 & 2.665 & 797.667 \\
\hline $16-10-10 / 19: 05-19: 10$ & 0.019 & 10.806 & 48.495 & $9,451.304$ & 2.594 & 265.243 & 1.072 & 148.461 & 0.726 & 49.174 & 2.444 & 11.242 & -3.219 & -1.855 & -5.317 & -1.755 & 4.380 & 515.000 \\
\hline $16-10-16 / 19: 15-19: 20$ & 0.010 & 2.132 & 31.153 & $4,993.229$ & 3.109 & 353.942 & 1.365 & 117.251 & 0.841 & 54.955 & 3.088 & 11.085 & -2.730 & 1.180 & -1.388 & -1.723 & 3.363 & 511.000 \\
\hline $16-10-24 / 17: 00-17: 05$ & 0.013 & 10.196 & 35.503 & $14,807.722$ & 3.488 & 396.836 & 1.874 & 218.140 & 0.908 & 95.626 & 5.228 & 10.248 & -3.330 & -4.968 & -2.340 & -1.521 & 2.084 & 615.167 \\
\hline $16-10-25 / 19: 35-19: 40$ & 0.110 & 3.711 & 48.360 & $11,192.526$ & 14.878 & 286.085 & 7.982 & 129.610 & 2.818 & 85.624 & 3.871 & 10.630 & -1.845 & -7.228 & -2.135 & -1.263 & 4.426 & 533.833 \\
\hline $16-10-26 / 20: 30-20: 35$ & 0.077 & 1.376 & 40.532 & $4,397.794$ & 8.588 & 268.889 & 4.054 & 166.867 & 1.901 & 96.013 & 3.352 & 10.428 & -1.329 & -3.675 & 1.825 & -1.085 & 2.458 & 611.667 \\
\hline $16-10-27 / 09: 55-10: 00$ & 0.039 & 1.300 & 29.068 & $4,507.753$ & 9.425 & 147.744 & 4.256 & 31.553 & 1.359 & 7.836 & 7.079 & 5.093 & -3.753 & -1.590 & 1.937 & -1.040 & 2.018 & 602.000 \\
\hline $16-10-27 / 13: 20-13: 25$ & 0.007 & 4.775 & 28.087 & $10,389.690$ & 3.113 & 184.251 & 1.421 & 62.515 & 0.938 & 26.536 & 6.941 & 8.013 & -4.363 & -4.160 & 1.272 & -0.552 & 1.482 & 857.000 \\
\hline $16-10-30 / 10: 38-10: 42$ & 0.041 & 6.901 & & $19,376.901$ & 9.000 & & 5.035 & 42.561 & 1.908 & 7.614 & 1.540 & 5.815 & & & 5.597 & & 1.958 & \\
\hline $16-10-30 / 14: 19-14: 24$ & 0.027 & 2.302 & 27.364 & $6,457.850$ & 6.944 & 125.662 & 3.486 & 53.454 & 1.281 & 31.814 & 7.037 & 8.650 & -3.938 & -2.507 & 5.683 & -0.082 & 1.280 & 689.500 \\
\hline $16-11-01 / 14: 25-14: 30$ & 0.026 & 4.780 & 35.876 & $12,501.479$ & 8.350 & 89.432 & 4.044 & 43.039 & 1.145 & 25.107 & 7.238 & 8.656 & -3.735 & -5.298 & 3.512 & -0.010 & 1.678 & 825.500 \\
\hline $16-11-03 / 15: 00-15: 05$ & 0.013 & 8.588 & 34.976 & $11,027.917$ & 5.616 & 133.266 & 1.749 & 70.526 & 0.731 & 32.264 & 7.255 & 8.917 & & -3.302 & 1.390 & 0.455 & 1.705 & 538.833 \\
\hline 016-11-10/18:00-18:05 & 0.099 & 10.036 & 42.347 & $17,276.490$ & 15.741 & 978.148 & 10.028 & 521.538 & 4.633 & 198.498 & 6.326 & 9.367 & -1.185 & 4.717 & -12.147 & 1.289 & 3.937 & 505.833 \\
\hline 105 P & 0.140 & 12.607 & 56.746 & $21,853.488$ & $10.0 \angle 2$ & 210.170 & 6.624 & 131.102 & 1.690 & 65.935 & 6.552 & 7.823 & 0.411 & -4.878 & 8.120 & 1.738 & 4.230 & 980.333 \\
\hline $16-12-09 / 17: 55-18: 00$ & & 14.206 & 48.722 & $27,833.406$ & & 400.408 & 4.492 & 104.636 & 1.806 & & 7.119 & 5.964 & 1.317 & 2.483 & -4.498 & 2.095 & 2.928 & 867.0 \\
\hline $010-12-11 / 10.05-10.15$ & 0.000 & 8.925 & 31.344 & $15,876.745$ & 2.030 & 103.050 & 1.090 & 91.379 & 0.773 & 58.836 & 8.833 & 5.651 & 0.869 & 1.319 & -3.365 & 2.790 & 2.884 & 561.364 \\
\hline $16-12-21 / 16 \cdot 40-16$ & 040 & 26.924 & 53.825 & $57,299.640$ & 4.415 & $510.4 \angle 0$ & 2.647 & 122.863 & 1.432 & 47.428 & 7.969 & 4.424 & 1.650 & -8.322 & 6.352 & 3.475 & 5.648 & $1,652.833$ \\
\hline $16-12-26 / 15: 00-15: 05$ & 0.013 & 12.586 & 34.978 & $27,348.612$ & 3.768 & 476.602 & 1.189 & 67.514 & 0.682 & 15.257 & 9.381 & 3.479 & 1.533 & -4.730 & 0.708 & 4.402 & 2.380 & 793.000 \\
\hline
\end{tabular}

